\title{
A Rubik's Snake with General Rotation Angles
}

\section{Songming Hou ${ }^{1, *}$, Scott Atkins ${ }^{2}$, Yu Chen ${ }^{3}$}

${ }^{1}$ Program of Math\&Stats and Center of Applied Physics, Louisiana Tech University, Ruston, LA 71272

${ }^{2}$ Louisiana School for Math, Science, and the Arts, Natchitoches, LA 71457

${ }^{3}$ Program of Math\&Stats, Louisiana Tech University, Ruston, LA 71272

*Corresponding author: shou@1atech.edu

\begin{abstract}
A Rubik's Snake is a toy that has been around for 40 years. It is a system of serial chain. It traditionally only allows rotations of its pieces in 90 degree increments. In this paper we are not going to restrict ourselves to these limited number of incremental rotations. We prove a theorem about the sum of rotations of a Rubik's Snake that closes on itself. We present an alternative representation for the Rubik's Snake, different from the standard representation that the toy has. We will use matrices to study the rotations of the pieces of the Rubik's Snake. In particular we will study two cases where all of the pieces of a Rubik's Snake have the same rotation angle, proving a theorem comparing the first and last pieces of a Rubik's Snake.
\end{abstract}

Keywords: Rubik's Snake, robot design 


\section{Introduction}

The Rubik's Snake is a toy [1] that was invented by Prof. Erno Rubik in 1981 [2,3]. It typically consists of 24 right isosceles triangular prisms (what we are calling blocks) that, except for the first and last block, are connected to two other blocks at the centers of the square faces. In this paper we will not be restricted to 24 blocks.

In [2] Rubik states that "The snake is not a problem to be solved; it offers infinite possibilities of combination .... lifetime is not sufficient to realize all of its possibilities." Rubik's original design of the toy allowed for rotations of $0^{\circ}$ ( or $360^{\circ}$ ), $90^{\circ}, 180^{\circ}$, or $270^{\circ}$ of one block relative to an adjacent block. In this paper we are going to allow any rotation angle between $0^{\circ}$ and $360^{\circ}$ which will give even more possibilities than Rubik’s original concept.

The Rubik's Snake has been used as a tool for the study of protein folding [4,5] and for the construction of reconfigurable modular robots [6-8]. It can be viewed as a serial chain [9]. More applications of robots can be found in [10,11]. Some ideas in the study of Rubik's Snake such as the use of rotation matrix is also used in rigid Origami folding [12,13]. In previous papers, strategies have been given for the design of a Rubik's Snake [14], and some mathematical problems concerning a Rubik's Snake have been studied [15]. Rotations that are not $0^{\circ}$ (or $360^{\circ}$ ), $90^{\circ}, 180^{\circ}$, or $270^{\circ}$ are mentioned in [14] but not much theoretical work is presented. On the other hand, [15] has quite some theoretical work but is only concerned with integer multiple of 90 degree rotations. Also, in [16], theorems about palindromic, periodic and Möbius Rubik's snakes were proved but most of them were concerned with integer multiple of 90 degree rotations. As was mentioned earlier, in this paper we are going to allow any rotation between $0^{\circ}$ and $360^{\circ}$. Theoretical work and applications are both included.

In this paper, we first define what is meant by the degree of rotation of a block. We then describe a tube representation for the snake and compare it to the block representation. We also develop a diagonalized rotation matrix for the rotation of one block relative to the previous block. Finally, we apply our results to the study of a Rubik's Snake with a constant degree of rotation and discuss the orientation and shift of the last block.

\section{Degree of Rotation of a Block of a Rubik's Snake}

Let $\theta$ be the angle of rotation between two blocks. As in [14] and [15], we define $d=\theta / 90^{\circ}$ to be the degree of this rotation. In a normal Rubik's Snake the degree of rotation is fixed to be 
either $0,1,2$, or 3 , corresponding to a rotation of $0^{\circ}, 90^{\circ}, 180^{\circ}$, or $270^{\circ}$, respectively. This definition makes it convenient to write sequences involving a lot of integer multiple of 90 degree rotations. In the current paper we are not going to restrict the rotation of the blocks to these four choices: we can have $d \in[0,4)$. For example, if we rotate it $45^{\circ}$, then the rotation will have degree 0.5 .

In [15], we proved that the sum of degrees of a Rubik's Snake closed loop with integer degrees of rotation must be an even number. In other words it is 0 or 2 in the mod 4 sense. Below we prove a theorem about the sum of degrees when the non-integer degrees of rotation are allowed. The idea is to construct a class of closed loops with special geometry with parameters that allow us to adjust the sum of degrees.

Theorem 2.1. For any real number $t \in[0,4)$, there exists a Rubik's Snake closed loop such that the sum of degrees, given by $S=\sum_{i=1}^{n} d_{i}, \bmod 4$ equals $t$.

Proof. To prove this theorem, we use the sequence $[d, 3,0,3, d-4 / n, 3,0,3]$, where $d \in[0,4)$, repeated $n$ times to form a closed loop. Our choice of $d$ is completely random: to get closed loops of different geometries, we simply need to vary the values of $d$ and $n$.

For blocks 2 through 9, each rotation in the sequence $[d, 3,0,3, d-4 / n, 3,0,3]$ is applied in order. This process is repeated for each successive grouping of eight blocks: $n$ times until there is a closed loop. For example, looking at Fig. 1, it can be seen that every grouping of eight blocks (starting with block 2) repeats the sequence with $d=\sqrt{3}-1$ and $n=12$.

By considering the projection onto a $2 \mathrm{D}$ plane, we could see that the difference in angle between the first and the fifth entry in the sequence $[d, 3,0,3, d-4 / n, 3,0,3]$ is equal to the difference in orientation angles for every eighth block (starting with block 2 ). If the $4 / n$ term were missing, every eighth block (starting with block 2) would have the same orientation angle and a closed loop would not be possible. Looking at Fig. 1 again, this means that blocks $2,10,18, \ldots$ would all point in the same direction.

Since the difference in orientation angles are the same for every eighth block (starting with block 2), the sum of these $n$ orientation angle differences must add to $2 \pi$ in order for the loop to be closed. This gives that each orientation angle difference must be $2 \pi / n$, which is equivalent to a degree of $4 / n$. This explains why we need the $4 / n$ term to make a closed loop. Calculating the sum of the degrees for our example sequence repeated $n$ times, we obtain $S=$ $2 d n+12 n-4$. Taking $S$ modulo 4 , we obtain $S \bmod 4=2 d n \bmod 4$. Obviously $S$ can be any real 
number in $[0,4)$ in the sense of mod 4 with the freedom to choose $d$ and $n$. This completes the proof.

Let us choose $d=\sqrt{3}-1$ and $n=12$ as a specific example. Using these choices, we obtain the closed loop given in Fig. 1 with $S \approx 157.56921938$ with $S \bmod 4 \approx 1.56921938$ being a real number in $[0,4)$.

We would make a remark that it can be seen from the proof that the period of a closed loop with general angle rotations can be any positive integer. This is different from the integer degree case in [15] where the period is proved to be no more than 4 .

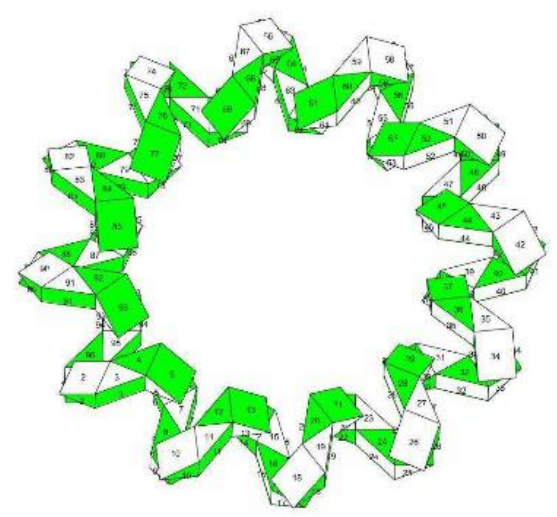

Fig. 1: Closed loop with 96 blocks. The sequence $[d, 3,0,3, d-4 / n, 3,0,3]$ has been repeated $n=12$ times with $d=\sqrt{ } \mathbf{3}-1$.

\section{Tube Representation of a Rubik's Snake}

We can consider each block of the Rubik's Snake as a small piece of a tube as shown in Fig. 2. We can study the Rubik's Snake using the tube representation instead of the block representation. This will allow us to see finer details.

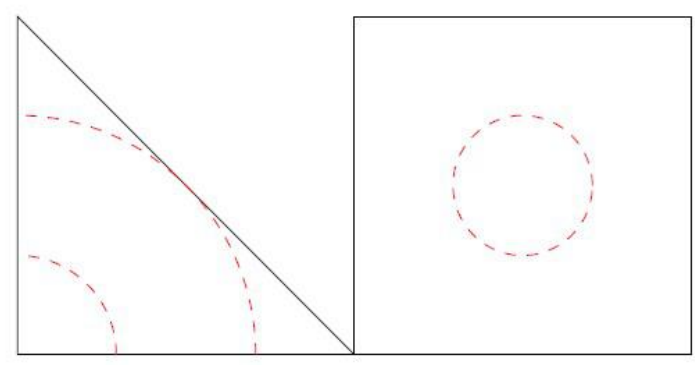

Fig. 2: The left figure shows the projection of the tube onto a triangular face. The right figure shows the tube on the entrance or exit square faces. 
In [15], we defined the trail an ant would take (subject to certain rules) while walking along a closed loop. We can apply this idea on a tube instead of the original block representation. If an ant stands on the surface of a tube and walks "straight" (to be more precise, the angle parameter on the cross section of the tube within one block is unchanged for the ant and the path is continuous within a block or between blocks) along the surface, it will eventually come back to the piece of tube on which it started. Between the $i$ th and the next block, the cross section angle parameter for the ant is changed by $\pi-\theta_{i}$, where $\theta_{i}$ is the rotation angle in radian measure between the $i$ th block and the next block. From Theorem 2.1, we know the sum of $\theta_{i}$ for one round of a closed loop could be any real number in $[0,2 \pi)$ when removing a multiple of $2 \pi$. This means the sum of $\pi-\theta_{i}$ could be any real number in $[0,2 \pi)$ when removing a multiple of $2 \pi$ as well. Eventually when the ant travels one round on a closed loop, the angle parameter on the cross section for the ant could be any real number in $[0,2 \pi)$ when removing a multiple of $2 \pi$. The ant might not come back to where it started due to the angle difference.

We see two examples of the tube representation side-by-side with its block representation. In

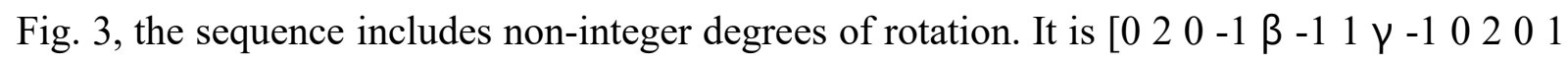

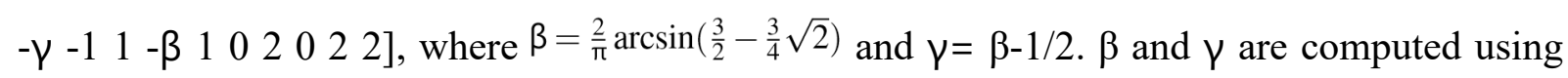
geometry to make this a closed loop. In [15], we proved that a closed loop with integer degrees of rotations must have even number of pieces, and if two blocks of the same Rubik's Snake share a cube, they must have the same color (assuming the regular coloring of the snake, i.e. alternated coloring). However, this example is a closed loop with an odd number of pieces ( 23 pieces), and it has two blocks sharing a cube that have different colors. The reason is non-integer degrees are included. In Fig. 4, only integer degrees of rotation are included in an example of a racing car shape.

We can see that the tube representation looks smooth compared to the original block representation. The irregularity of the non-integer degree rotation cannot be easily observed in the tube version. Also, some hidden details of a complicated path is revealed in the tube version: you can trace the path easier in the tube version of the racing car example than the original version. We proved earlier that the sum of degrees of a Rubik's Snake closed loop can be any real number in $[0,4)$ in the sense of mod 4 . This means the ant trail going one round can get back to any angle of the tube's cross section by choosing parameters in the sequence $[d, 3,0,3, d-4 / n, 3,0,3]$. In the racing car example, the ant would come back to the 
original angle on the tube with the sum of degrees being a multiple of 4 . In the example with odd pieces, the ant also comes back to the original angle because when summing up $\pi-\theta_{i}$, the odd piece would contribute an extra $\pi$ compared with the even piece case, and this effect cancels with the effect from the extra 2 in $4 k+2$ form (note that the sum of degrees in the sequence with 23 pieces is 10). It is not really a Möbius strip even with the sum of degrees of $4 k+2$ because the assumption of integer degree in the theorem in [15] is violated here.
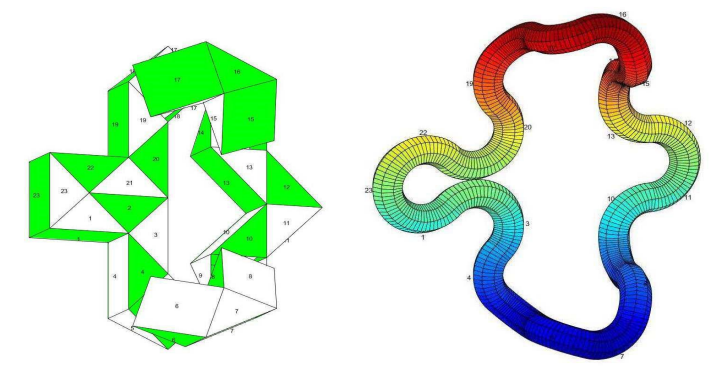

Fig. 3: Closed loop with 23 blocks on left. Tube representation on right.
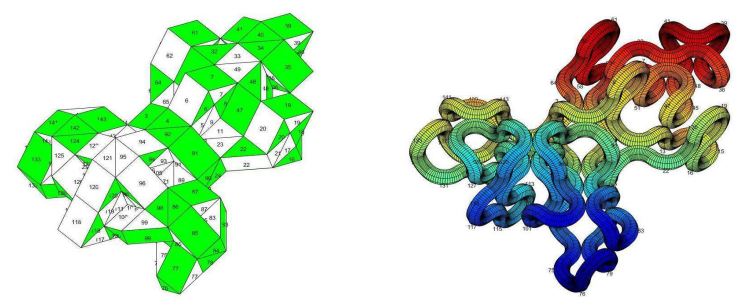

Fig. 4: Closed loop with 144 blocks on left. Tube representation on right.

\section{Matrix Representation of a Rubik's Snake}

Let $\alpha$ be the angle between the longest sides of two adjacent blocks, and let $\theta$ be the angle of rotation between two adjacent square faces. For the purposes of finding a relationship between $\alpha$ and $\theta$, we define the coordinate system relative to the first block as shown in Fig. 5, where $\vec{v}_{1}$ and $\vec{v}_{2}$ are the vectors for the longest sides of the adjacent blocks and are given by

$$
\vec{v}_{1}=\left(\begin{array}{l}
1 \\
1 \\
0
\end{array}\right) \quad \vec{v}_{2}=\left(\begin{array}{l}
1 \\
1 \\
0
\end{array}\right)
$$




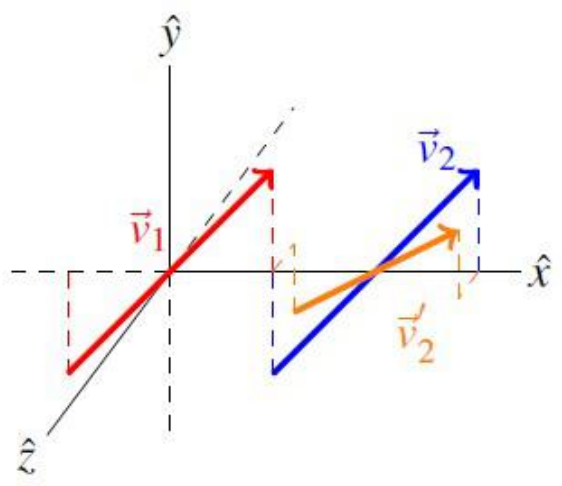

Fig. 5: View of blocks 1 and 2 before and after rotation of block $2 . \vec{v}_{2}{ }^{\prime}$ is what $\vec{v}_{2}$ becomes after a rotation of $\theta$ about the $x$-axis, and $\alpha$ is the angle between $\vec{v}_{1}$ and $\vec{v}_{2}$,

and $\theta$ is the angle of rotation about the $\mathrm{x}$-axis with the rotation matrix being

$$
R_{x}(\theta)=\left(\begin{array}{ccc}
1 & 0 & 0 \\
0 & \cos \theta & -\sin \theta \\
0 & \sin \theta & \cos \theta
\end{array}\right)
$$

Let us rotate $\vec{v}_{2}$ about the X-axis to obtain

$$
\vec{v}_{2}^{\prime}=\left(\begin{array}{ccc}
1 & 0 & 0 \\
0 & \cos \theta & -\sin \theta \\
0 & \sin \theta & \cos \theta
\end{array}\right)\left(\begin{array}{l}
1 \\
1 \\
0
\end{array}\right)=\left(\begin{array}{c}
1 \\
\cos \theta \\
\sin \theta
\end{array}\right)
$$

Using $\vec{v}_{1} \cdot \quad \vec{v}_{2}^{\prime}=\left\|\vec{v}_{1}\right\|\left\|\vec{v}_{2}^{\prime}\right\| \cos \alpha$ with $\vec{v}_{1} \cdot \vec{v}_{2}^{\prime}=1+\cos \theta$, we have the following relationship between $\theta$ and $\alpha$ :

$$
\theta=\arccos (2 \cos \alpha-1)
$$

\subsection{Rotation Matrix}

We can define the rotation matrix relating the neighboring snake blocks as was done in [14] by

$$
\begin{aligned}
R(\theta) & =R_{z}(\pi / 2) R_{x}(\pi-\theta) \\
& =\left(\begin{array}{lll}
0 & -1 & 0 \\
1 & 0 & 0 \\
0 & 0 & 1
\end{array}\right)\left(\begin{array}{ccc}
1 & 0 & 0 \\
0 \cos (\pi-\theta) & -\sin (\pi-\theta) \\
0 \sin (\pi-\theta) & \cos (\pi-\theta)
\end{array}\right) \\
& =\left(\begin{array}{ccc}
0 & 2 \cos \alpha-1 & \sqrt{1-(2 \cos \alpha-1)^{2}} \\
1 & 0 & 0 \\
0 & \sqrt{1-(2 \cos \alpha-1)^{2}} & -(2 \cos \alpha-1)
\end{array}\right)
\end{aligned}
$$


The formula can be viewed as a special case of the Denavit-Hartenberg convention [9] after the translation is separated from the rotation.

We need to find the eigenvalues for $R(\theta)$ by solving the characteristic equation for $R(\theta)$ :

$$
\begin{aligned}
0 & =\lambda^{3}+(2 \cos \alpha-1) \lambda^{2}-(2 \cos \alpha-1) \lambda-1 \\
& =(\lambda-1)\left(\lambda+e^{-i \alpha}\right)\left(\lambda+e^{i \alpha}\right)
\end{aligned}
$$

These eigenvalues along with their corresponding eigenvectors are listed below, where the * stands for complex conjugate:

$$
\begin{array}{ll}
\lambda_{1}=1 & \vec{v}_{1}=\left(\begin{array}{c}
2 \cos \alpha \\
2 \cos \alpha \\
\sqrt{1-(2 \cos \alpha-1)^{2}}
\end{array}\right) \\
\lambda_{2}=-e^{-i \alpha} & \vec{v}_{2}=\left(\begin{array}{c}
e^{-i \alpha} \\
-1 \\
\frac{\sqrt{1-(2 \cos \alpha-1)^{2}}}{1-e^{i \alpha}}
\end{array}\right) \\
\lambda_{3}=\left(\lambda_{2}\right)^{*} & \vec{v}_{3}=\left(\vec{v}_{2}\right)^{*}
\end{array}
$$

Note: If $\theta=\pi$ (i.e. $\alpha=\pi / 2)$, then $\vec{v}_{1}=(0,0,1)$ not $(0,0,0)$.

Now we can diagonalize $R(\theta)$ as follows: $R(\theta)=P \Lambda P^{-1}$ where $P=\left(\vec{v}_{1}, \vec{v}_{2}, \vec{v}_{3}\right)$ and

$$
\Lambda=\left(\begin{array}{ccc}
\lambda_{1} & 0 & 0 \\
0 & \lambda_{2} & 0 \\
0 & 0 & \lambda_{3}
\end{array}\right)=\left(\begin{array}{ccc}
1 & 0 & 0 \\
0 & -e^{-i \alpha} & 0 \\
0 & 0 & -e^{i \alpha}
\end{array}\right)
$$

In the next two subsections we will be looking at examples where all blocks have the same $\alpha$ : $\alpha=\pi / m$ and $\alpha=2 \pi / m$, where $m$ is an integer. Further restrictions will be placed upon $m$ in the corresponding subsections.

\subsection{Blocks with $\alpha=\pi / \mathrm{m}$}

In this subsection we are going to look at what happens if all blocks have the same degree of rotation. In particular all blocks will have $\alpha=\pi / m$.

Theorem 4.1. In a Rubik's Snake with $\mathrm{m}$ ( $\mathrm{m} \geq 3, \mathrm{~m}$ odd) junctions or $2 \mathrm{~m}$ ( $\mathrm{m} \geq 2, \mathrm{~m}$ even) junctions and with every junction having degree $d=2 \arccos (2 \cos (\pi / m)-1) / \pi$ (i.e. $\alpha=\pi / m)$, then the orientations of the "start" and "end" blocks are the same.

Proof. Let "a" denote the orientation of a random block. Proving that the "start" and "end" blocks are the same orientation is equivalent to showing that $a R(\theta)^{n}=a$, where $n=m(m \geq$ 3 , $\mathrm{m}$ odd) or $n=2 m$ ( $m \geq 2, \mathrm{~m}$ even). This means that we need to show that $R(\theta)^{n}=I$ for $n=m$ 
( $m \geq 3$, m odd) or $n=2 m$ ( $m \geq 2$, m even).Using $\alpha=\pi / m$ in the diagonalized $R(\theta)$, we have $R(\theta)$ $=P \Lambda P^{-1}$, where $P=\left(\vec{v}_{1}, \vec{v}_{2}, \vec{v}_{3}\right)$ and

$$
\Lambda=\left(\begin{array}{ccc}
1 & 0 & 0 \\
0 & -e^{-i \frac{\pi}{m}} & 0 \\
0 & 0 & -e^{i \frac{\pi}{m}}
\end{array}\right)=\left(\begin{array}{ccc}
1 & 0 & 0 \\
0 & e^{i\left(\frac{m-1}{m}\right) \pi} & 0 \\
0 & 0 & e^{-i\left(\frac{m-1}{m}\right) \pi}
\end{array}\right)
$$

Using this we have $R(\theta)^{n}=P \Lambda^{n} P^{-1}$, where

$$
\Lambda^{n}=\left(\begin{array}{ccc}
1 & 0 & 0 \\
0 e^{i\left(\frac{m-1}{m}\right) n \pi} & 0 \\
0 & 0 & e^{-i\left(\frac{m-1}{m}\right) n \pi}
\end{array}\right)
$$

If $n=m(m \geq 3, \mathrm{~m}$ odd $)$ or $n=2 m(m \geq 2, \mathrm{~m}$ even $)$, then we have $\Lambda^{n}=I$. This gives that $R(\theta)^{n}=$ $I$ for $n=m(m \geq 3, \mathrm{~m}$ odd $)$ or $n=2 m$ ( $m \geq 2$, m even). This completes the proof.

Since we have shown that the "start" and "end" blocks have the same orientation for $n=m$ ( $m$ $\geq 3, \mathrm{~m}$ odd) or $n=2 m$ ( $m \geq 2$, m even) for $\alpha=\pi / m$, we would like to know what the shift in position of the "end" block is. We have seen in [14] that to find the location of the $(n+1)$ th block we apply an iterative technique:

$$
\vec{r}_{n+1}=\vec{r}_{n}+(1,0,0) \underline{D}_{n} \quad \underline{D}_{n+1}=R(\theta) \underline{D}_{n}
$$

where $\vec{r}_{1}=(0,0,0), \underline{D}_{1}=I, R(\theta)=P \wedge P^{-1}$, and

$$
\Lambda=\left(\begin{array}{ccc}
1 & 0 & 0 \\
0 & e^{i\left(\frac{m-1}{m}\right) \pi} & 0 \\
0 & 0 & e^{-i\left(\frac{m-1}{m}\right) \pi}
\end{array}\right)
$$

$\underline{D}_{i}$ represents the local coordinate system that is attached to the ith block. When a block is rotated, the coordinate system is also rotated. $\vec{r}_{i}$ represents the position of the ith block. Performing all of the iterative steps, we derive a formula for the position of the $(n+1)$ th block relative to the "starting" block:

$$
\vec{r}_{n+1}=(1,0,0) R_{n-1}
$$

where $R_{n-1}=P \Lambda_{n-1} P^{-1}$ and

$$
\Lambda_{n-1}=I+\Lambda+\Lambda^{2}+\ldots+\Lambda^{n-1} .
$$


This sum is diagonal, and for $n=m(m \geq 3, \mathrm{~m}$ odd $)$ and $n=2 m(m \geq 2, \mathrm{~m}$ even $)$ :

$$
\begin{array}{r}
\Lambda_{n-1}^{(1,1)}=1+\sum_{k=1}^{n-1} 1^{k}=n \\
\Lambda_{n-1}^{(2,2)}=1+\sum_{k=1}^{n-1} e^{\frac{i(m-1) k \pi}{m}}=1+\left\{\frac{e^{\frac{i(m-1) \pi}{m}}-e^{\frac{i(m-1) n \pi}{m}}}{1-e^{\frac{i(m-1) \pi}{m}}}\right\}=0 \\
\Lambda_{n-1}^{(3,3)}=1+\sum_{k=1}^{n-1} e^{\frac{-i(m-1) k \pi}{m}}=1+\left\{\frac{e^{\frac{-i(m-1) \pi}{m}}-e^{\frac{i(m-1) n \pi}{m}}}{1-e^{\frac{-i(m-1) \pi}{m}}}\right\}=0
\end{array}
$$

Then we have for $n=m(m \geq 3, \mathrm{~m}$ odd $)$ and $n=2 m$ ( $m \geq 2, \mathrm{~m}$ even):

$$
\wedge_{n-1}=\left(\begin{array}{ccc}
n & 0 & 0 \\
0 & 0 & 0 \\
0 & 0 & 0
\end{array}\right)
$$

Using this and $P=\left(\vec{v}_{1}, \vec{v}_{2}, \vec{v}_{3}\right)$, we can calculate $R_{n-1}=P \Lambda_{n-1} P^{-1}$ :

$$
R_{n-1}=\left(\begin{array}{ccc}
2 \delta \cos \frac{\pi}{m} & 2 \delta \cos \frac{\pi}{m} & \delta \sin \theta \\
2 \delta \cos \frac{\pi}{m} & 2 \delta \cos \frac{\pi}{m} & \delta \sin \theta \\
\delta \sin \theta & \delta \sin \theta & 2 \delta\left(1-\cos \frac{\pi}{m}\right)
\end{array}\right)
$$

where $\delta=n /(2+2 \cos (\pi / m))$ and $\theta=\arccos (2 \cos (\pi / m)-1)$. Using Eq. 13, we can calculate the shift

$$
\begin{aligned}
S_{n} & =(1,0,0) R_{n-1} \\
& =\left(\frac{n \cos \frac{\pi}{m}}{1+\cos \frac{\pi}{m}} \frac{n \cos \frac{\pi}{m}}{1+\cos \frac{\pi}{m}} \frac{n \sqrt{1-\left(2 \cos \frac{\pi}{m}-1\right)^{2}}}{2\left(1+\cos \frac{\pi}{m}\right)}\right)
\end{aligned}
$$

$S_{n}$ represents the shift in position after rotation of the nth junction, about which the $(\mathrm{n}+1)$ th block rotates. Substituting $n=m(m \geq 3, \mathrm{~m}$ odd $)$ or $n=2 m$ ( $m \geq 2, \mathrm{~m}$ even), we can calculate the shifts for odd or even $\mathrm{m}$.

As two trivial examples of this, we are going to look at $m=3(n=m=3$, which means four blocks) and $m=2(n=2 m=4$, which means five blocks). For the $m=3$ case $d=$ $2 \arccos (2 \cos (\pi / m)-1) / \pi=1$ and $S_{3}=(1,1,1)$. Taking the starting block at the origin and making only 90 turns, the fourth block will end up at the position $(1,1,1)$. For the $m=2$ case $d=$ $2 \arccos (2 \cos (\pi / m)-1) / \pi=2$ and $S_{4}=(0,0,0)$. Taking the starting block at the origin and making only $180^{\circ}$ turns, the fifth block will end up at the same position of the starting block. In the next subsection, we will present two non-trivial examples.

\subsection{Blocks with $\alpha=2 \pi / \mathrm{m}$}

Now we are going to consider a Rubik's Snake where the degree for each rotation is $d=$ $2 \arccos (2 \cos (2 \pi / m)-1) / \pi$. If $m$ is even (i.e. $m=2 k$ ), then we get the results discussed 
previously, looking at whether $k$ is odd or even. Therefore, we need only consider the case where $m$ is odd. In order to have $\alpha=2 \pi / m$ be in the range $0 \leq \alpha \leq \pi / 2$, we need to have $m \geq 5$. For $\alpha=2 \pi / m$, we have for $\Lambda$ :

$$
\Lambda=\left(\begin{array}{ccc}
1 & 0 & 0 \\
0 & e^{i\left(\frac{m-2}{m}\right) \pi} & 0 \\
0 & 0 & e^{-i\left(\frac{m-2}{m}\right) \pi}
\end{array}\right)
$$

In order for the "start" and "end" blocks to have the same orientation, $2 m$ junctions are needed since $\Lambda^{2 m}=I$. Going through a similar process as before, we find that the shift in position (given $\alpha=2 \pi / m, 2 m$ junctions, $m$ odd, and $m \geq 5$ ) is

$$
S_{2 m}^{\prime}=\left(\frac{2 m \cos \frac{2 \pi}{2 \pi}}{1+\cos \frac{2 \pi}{m}} \frac{2 m \cos \frac{2 \pi}{m}}{1+\cos \frac{2 \pi}{m}} \frac{2 m \sqrt{1-\left(2 \cos \frac{2 \pi}{m}-1\right)^{2}}}{2\left(1+\cos \frac{2 \pi}{m}\right)}\right)
$$

Now we present two nontrivial examples. Fig. 6 shows the snake sequence $2 \arccos (2 \cos (\pi / 5)-1) / \pi$ repeated times on the left part, and then $2 \arccos (2 \cos (2 \pi / 5)-1) / \pi$ repeated 10 times on the right. Note that the first and the sixth blocks on the left figure have the same orientation as we proved in the previous subsection, and the first and the eleventh blocks on the right figure have the same orientation as we proved in this subsection. The shifts also agree with the results produced by our formulas. As a remark, some local collisions between the $i$ th and the $(i+2)$ th blocks occur, but these can be avoided if we use the tube representation of the snake discussed earlier because it is the rotation of the corners of the cross sectional square faces that leads to the problem and because the tube cross section is a circle with rotation invariance.

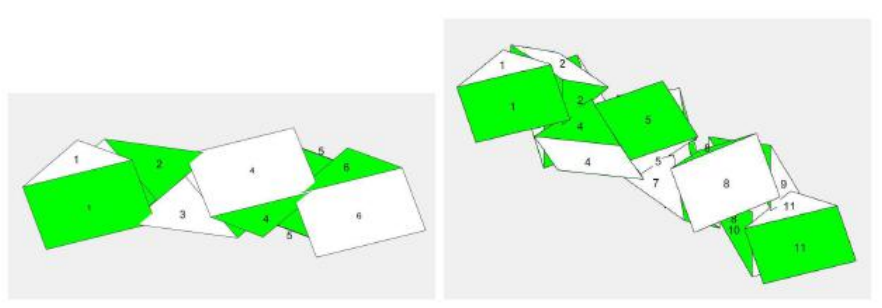

Fig. 6: Two nontrivial examples of the first and last block having exactly the same orientation.

\section{Conclusion}

We have generalized a Rubik's Snake to have any angle of rotation between $0^{\circ}$ and $360^{\circ}$, proving a theorem about the sum of degrees of rotation for a snake that is a closed loop with 
an arbitrary number of blocks. We also looked at an alternative picture (i.e. the tube representation) for the Rubik's Snake that allows us to see finer details that cannot be seen with the block representation. We used matrices to prove a theorem about the orientation of the ending block of the Rubik's Snake relative to the beginning block where all blocks had the same rotation angle and to derive the shift in position of the ending block relative to the beginning block in this case. The Rubik's Snake was originally meant to only rotate integer multiples of $90^{\circ}$ for each joint. Once it is generalized to allow general angles, it is easy to get lost. Fortunately, mathematical theories can be applied to help us to keep track and design shapes.

\section{Data Availability}

The data that support the findings of this study are available from the corresponding author upon reasonable request.

\section{Acknowledgment}

We thank Dr. Zilong Li and Dr. Jianning Su for interesting discussions. S. Hou's research is partially supported by the Walter Koss Endowed Professorship. The title of the professorship is made available through the State of Louisiana Board of Regents Support Funds.

\section{References}

[1] Fiore, A., 1981. Shaping Rubik's Snake. Penguin Books, Harmondsworth, Middlesex, England.

[2] Fenyvesi, C., 1981. "Rubik's snake of 'infinite possibilities"'. The Washington Post.

[3] Jensen, G., 1981. "Now meet Rubik's snake -'bigger than Rubik's cube!”’. United Press International.

[4] Iguchi, K., 1998. "A toy model for understanding the conceptual framework of protein folding: Rubik's magic snake model”. Mod. Phys. Lett. B, 12(13), p. 499-506.

[5] Iguchi, K., 1999. "Exactly solvable model of protein folding: Rubik's magic snake model". Int. J. Mod. Phys. B, 13(4), pp. 325-361. 
[6] Ding, X., Lu, S., and Yang, Y., 2011. "Configuration transformation theory from a chaintype reconfigurable modular mechanism-Rubik's snake". The 13th World Congress in Mechanism and Machine Science.

[7] Zhang, X., and Liu, J., 2016. "Prototype design of a Rubik snake robot", Vol. 36 of Mechanisms and Machine Science.

[8] Liu, J., Zhang, X., Zhang, K., Dai, J. S., Li, S., and Sun, Q., 2019. "Configuration analysis of a reconfigurable Rubik's snake robot". Proceedings of the Institution of Mechanical Engineers, Part C: Journal of Mechanical Engineering Science, 233(9), pp. $3137-3154$.

[9] Detmvit, J., and Hartenberg, R., 1955. "A kinematic notation for lower-pair mechanisms based on matrices". ASME Journal of Applied Mechanics, pp. 215-221.

[10] Yim, M., Roufas, K., Duff, D., Zhang, Y., Eldershaw, C., and Homans, S., 2003. "Modular reconfigurable robots in space applications". Autonomous Robots, 14(2-3), pp. $225-237$.

[11] Zhang, X., Liu, J., Feng, J., Liu, Y., and Ju, Z., 2020. "Effective capture of nongraspable objects for space robots using geometric cage pairs". IEEE/ASME Transactions on Mechatronics, 25(1), pp. 95-107.

[12] Hull, T. C., and Belcastro, S.-m., 2002. "Modelling the folding of paper into three dimensions using affine transformations". Linear Algebra and its applications, 348(1-3), pp. 273-282.

[13] Tachi, T., 2009. "Simulation of rigid origami”. Origami, 4(08), pp. 175-187.

[14] Li, Z., Hou, S., and Bishop, T., 2020. "Computational design and analysis of a magic snake". J. Mech. Rob., 12(5), p. 054501.

[15] Hou, S., Chen, Y., and Li, Z., 2021. "Some mathematical problems related to the Rubik's snake". J. Mech. Rob., 13(1), p. 014502.

[16] Hou, S., Su, J., and Chen, Y., 2021. "Palindromic, periodic and Möbius Rubik's snakes". International Robotics and Automation Journal, 7(3), pp. 84-88. 